\title{
Effects of intraocular lenses with different diopters on chromatic aberrations in human eye models
}

\author{
Hui Song, Xiaoyong Yuan and Xin Tang*
}

\begin{abstract}
Background: In this study, the effects of intraocular lenses (IOLs) with different diopters (D) on chromatic aberration were investigated in human eye models, and the influences of the central thickness of IOLs on chromatic aberration were compared.

Methods: A Liou-Brennan-based IOL eye model was constructed using ZEMAX optical design software. Spherical IOLs with different diopters (AR40e, AMO Company, USA) were implanted; modulation transfer function (MTF) values at $3 \mathrm{~mm}$ of pupil diameter and from 0 to out-of-focus blur were collected and graphed.

Results: MTF values, measured at $555 \mathrm{~nm}$ of monochromatic light under each spatial frequency, were significantly higher than the values measured at 470 to $650 \mathrm{~nm}$ of polychromatic light. The influences of chromatic aberration on MTF values decreased with the increase in IOL diopter when the spatial frequency was $\leq 12 \mathrm{c} / \mathrm{d}$, while increased effects were observed when the spatial frequency was $\geq 15 \mathrm{c} / \mathrm{d}$. The MTF values of each IOL eye model were significantly lower than the MTF values of the Liou-Brennan eye models when measured at $555 \mathrm{~nm}$ of monochromatic light and at 470 to $650 \mathrm{~nm}$ of polychromatic light. The MTF values were also found to be increased with the increase in IOL diopter.

Conclusion: With higher diopters of IOLs, the central thickness increased accordingly, which could have created increased chromatic aberration and decreased the retinal image quality. To improve the postoperative visual quality, IOLs with lower chromatic aberration should be selected for patients with short axial lengths.
\end{abstract}

Keywords: Intraocular lens, Computer simulation, Diopter, Chromatic aberration, Modulation transfer function

\section{Background}

In polychromatic light, the retinal image quality is affected by interactions between monochromatic and chromatic aberrations. Some study had shown that ocular longitudinal chromatic aberration (LCA) was low intersubject variability and the LCA was independence of the presence of high order aberration [1]. Chromatic aberration has attracted increasing attention in intraocular lenses (IOLs) implantation for cataract surgeries. Recent studies have demonstrated that correcting monochromatic aberrations and chromatic aberrations at the same time could improve visual acuity and contrast

\footnotetext{
*Correspondence: tangprofessor@aliyun.com

Tianjin Eye Hospital, Tianjin Key Laboratory of Ophthalmology and Vision Science, Clinical College of Ophthalmology, Tianjin Medical University, No. 4 Gansu Rd, Heping District, Tianjin 300020, China
}

sensitivity [2-4]. Applegate et al. [5] also demonstrated that correction of spherical aberrations could increase the contrast sensitivity of the retina by 12 -fold, and correcting chromatic aberration could increase contrast sensitivity by 5 -fold, while correcting both spherical and chromatic aberrations simultaneously could strikingly increase contrast sensitivity by 25 -fold.

In Weeber's study, subjects were implanted with an IOL correcting both LCA and spherical aberration (SA) in one eye and an IOL correcting only SA in the fellow eye. Although this study included a small number of subjects, it showed a tendency for better visual performance in the eyes where both aberrations were corrected [6]. Considering that the LCA causes a substantial defocus over the visible range of about 2 diopters (D), the additional correction of this aberration should 
further improve visual quality even considering the protective mechanisms [7]. Perez-Merino et al. have reported the chromatic difference of focus in two groups of pseudophakic eyes implanted with IOLs of different materials, and found statistical difference, consistent with Abbe number of IOL materials in the $532-787 \mathrm{~nm}$ range [8].

Chromatic aberration is not only associated with Abbe number, which is widely known by researchers, but some other factors, including IOL shapes (thickness and radii of curvature) and the value of the refractive index material, could also affect chromatic aberrations [9]. Investigating the effects of IOLs with different diopters on chromatic aberrations in human eyes could help to further improve patients' visual quality as well as the design of IOLs [10].

In the present study, Liou-Brennan-based IOL eye models [11] were constructed using ZEMAX optical design software (ZEMAX Development Corporation, Bellevue, WA, USA), and IOLs were implanted to investigate the effects of IOLs with different diopters on the modulation transfer function (MTF) values in monochromatic and polychromatic light.

\section{Methods}

\section{Construction parameters of the Liou-Brennan eye model}

The Liou-Brennan eye model was used as the control (Table 1, Fig. 1), while for the treatment, another eye model, namely an IOL eye model that was constructed according to the process of constructing the control eye model, except for the gradient of the refractive index lens, was replaced by certain IOLs. All the study was approved by the Research Ethics Committee at the Tianjin Eye Hospital.

\section{Optical parameters of IOLs}

To simulate and investigate the retinal image quality of human eye models after implantation of IOLs with different diopters $(-10 \mathrm{D}, 0 \mathrm{D}, 10 \mathrm{D}, 20 \mathrm{D}$, and $30 \mathrm{D})$, traditional spherical IOLs AR40e, (Abbott Medical Optics, Santa Ana, CA, US) were used in the present study. The optical parameters of these IOLs are listed in Table 2.

\section{Construction of the eye models}

The construction of a physical model of the human eyes is based on the anatomical features of humans and on experimental results [12]. The Liou-Brennan eye model has been regarded as the most comprehensive and accurate human model that best fulfills the physiological status of human eyes [13]. The parameters of the LiouBrennan eye model were entered into the ZEMAX optical design software to construct models of natural and aphakic eyes. Then, IOLs with different diopters were implanted to replace the natural lenses (the posterior surface of the IOL coincided with the posterior surface of the natural lens) to construct the IOL eye model. The vitreous chamber depth was optimized until the out-offocus blur was 0 (indicating that parallel rays could focus on the retina). The transmission of light through the optical systems was simulated according to the raytracing theory (Fig. 2). The axial lengths of the IOLs with different diopters are listed in Table 2.

Modulation transfer function (MTF) is the difference between monochromatic and polychromatic MTF in the same space frequencies. With an IOL diopter increase, the optical zone increases in thickness, resulting in the optical path leading to increased color. A\% represents the magnitude of this change. The MTF values at $3 \mathrm{~mm}$ of pupil diameter for each of the IOLs were collected with monochromatic $(555 \mathrm{~nm})$ and polychromatic (470-650 nm) light under different spatial frequencies and were graphed. Yellow-green light with a wavelength of $555 \mathrm{~nm}$ was chosen as the monochromatic light in the present study, while for polychromatic light, we chose light with wavelengths ranging from 470 to $650 \mathrm{~nm}$ (including monochromatic light with wavelengths of $470,510,555,610$, and $650 \mathrm{~nm}$ and with weights of $0.091,0.503,1,0.503$, and 0.107 , respectively). All of the MTF values were collected using the ZEMAX Optical Design Program.

\section{Comparison of the MTF curves}

Origin software, version 7.0, was used for the data processing and graphing. The trends and difference were compared among the MTF curves that were constructed with the data collected under same conditions. Student's $t$ test was used to compare the differences between the 2

Table 1 Construction parameters of the Liou-Brennan eye model

\begin{tabular}{lllcl}
\hline Surface & Radius of curvature $(\mathrm{mm})$ & Asphericity & Thickness $(\mathrm{mm})$ & Refractive index $(555 \mathrm{~nm})$ \\
\hline 1 & 7.77 & -0.18 & 0.50 & 1.376 \\
2 & 6.40 & -0.60 & 3.16 & 1.336 \\
3 (pupil) & 12.40 & -0.94 & 1.59 & Grad A $^{\mathrm{a}}$ \\
4 & Infinity & - & 2.43 & Grad Pa \\
5 & -8.10 & +0.96 & 16.27 & 1.336 \\
\hline
\end{tabular}

${ }^{a}:$ Grad $A$ and $P$ represent different formulas for calculating gradient refractive index formula 


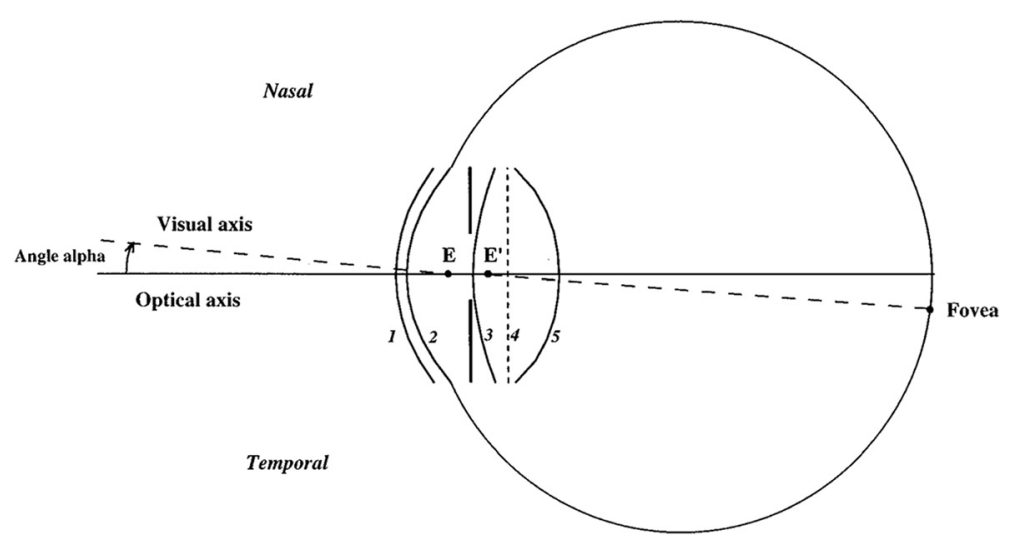

Fig. 1 Liou-Brennan eye models

groups. One-way ANOVA test was used to make comparisons between multiple groups ( $>3$ groups), and the Dunnett test was further used to compare different IOLs groups with the Liou group. Statistical significance was set at $p<0.05$.

\section{Results}

Comparisons of the MTF values of the IOL eye model under monochromatic and polychromatic light

MTF values at $3 \mathrm{~mm}$ of pupil diameter, measured at $555 \mathrm{~nm}$ of monochromatic light under each spatial frequency (ranging from 0 to $60 \mathrm{c} / \mathrm{d}$ ), were significantly higher than the values at $470-650 \mathrm{~nm}$ of polychromatic light (Fig. $3 \mathrm{a}-\mathrm{f}$ ). In addition, the differences between the MTF values that were measured under monochromatic (555 nm) and polychromatic (470-650 nm) light increased with the IOL diopter (i.e., the central thickness of the IOLs increased with the diopters, and the axial length decreased accordingly) (Fig. 3a-e). We also found that the A-value increased with the spatial frequencies (SFs), indicating the increased influence of chromatic aberration on MTF values (Table 3). In contrast, the Avalue decreased (indicating that the influence of chromatic aberration on MTF values decreased) with the increase of IOL diopters when the MTF values were measured at $3 \mathrm{~mm}$ of pupil diameter with spatial frequencies $\leq 12 \mathrm{c} / \mathrm{d}$. However, the A-value was found to be increased with the IOL diopters when the spatial frequencies were $\geq 15 \mathrm{c} / \mathrm{d}$ (Table 3 ).

\section{Comparisons of the MTF values between the IOL and Liou-Brennan eye models under monochromatic and polychromatic light}

The MTF values of most of the IOL eye models were significantly lower than those of the Liou-Brennan eye models when measured under monochromatic light (555 nm), with pupil diameter of $3 \mathrm{~mm}$ and spatial frequencies between 0 and $60 \mathrm{c} / \mathrm{d}$, and $\mathrm{F}$ values from 10 , $20,30,40,50$, and $60 \mathrm{c} / \mathrm{d}$ were $3.96,14.35,29.07,37.36$, $51.34,69.39$ with $p<0.05$ respectively. However, the MTF value of the IOL eye model with $30 \mathrm{D}$ was slightly higher than the MTF value of the Liou-Brennan eye model when measured with the spatial frequencies $\geq 50$ $\mathrm{c} / \mathrm{d}$. The MTF values of the IOL eye models increased with the IOL diopter (Fig. 4a). When measured under polychromatic light $(470-650 \mathrm{~nm})$, the MTF values of the IOL eye models were significantly lower than those of the Liou-Brennan eye models, with pupil diameter of $3 \mathrm{~mm}$ and spatial frequencies between 0 and $60 \mathrm{c} / \mathrm{d}$ with $\mathrm{F}$ values $3.38,8.91,17.15,28.69,47.45$, and 64.65 $(p<0.05)$, but the differences between the IOL and Liou-Brennan eye models were substantially less than those under monochromatic light $(555 \mathrm{~nm})$. We also

Table 2 Optical parameters and axial lengths of IOLs with different diopters

\begin{tabular}{lrcccc}
\hline $\begin{array}{l}\text { Diopters } \\
(\mathrm{D})\end{array}$ & $\begin{array}{l}\text { Refractive } \\
\text { index }\end{array}$ & $\begin{array}{l}\text { Curvature radius of the anterior surface } \\
(\mathrm{mm})\end{array}$ & $\begin{array}{l}\text { Central thickness } \\
(\mathrm{mm})\end{array}$ & $\begin{array}{l}\text { Curvature radius of the posterior surface } \\
(\mathrm{mm})\end{array}$ & $\begin{array}{l}\text { Axial length } \\
(\mathrm{mm})\end{array}$ \\
\hline+30.00 & 1.470 & 6.1951 & 1.224 & -15.7226 & 22.6597 \\
+20.00 & 1.470 & 11.6078 & 0.977 & -15.7226 & 24.9205 \\
+10.00 & 1.470 & 26.7716 & 0.760 & -26.7716 & 27.7042 \\
0.00 & 1.470 & 38.1000 & 0.533 & 38.0492 & 31.3168 \\
-10.00 & 1.470 & 50.8000 & 0.397 & 10.5918 & 36.4486 \\
\hline
\end{tabular}

$\mathrm{IOL}$ intraocular lens 


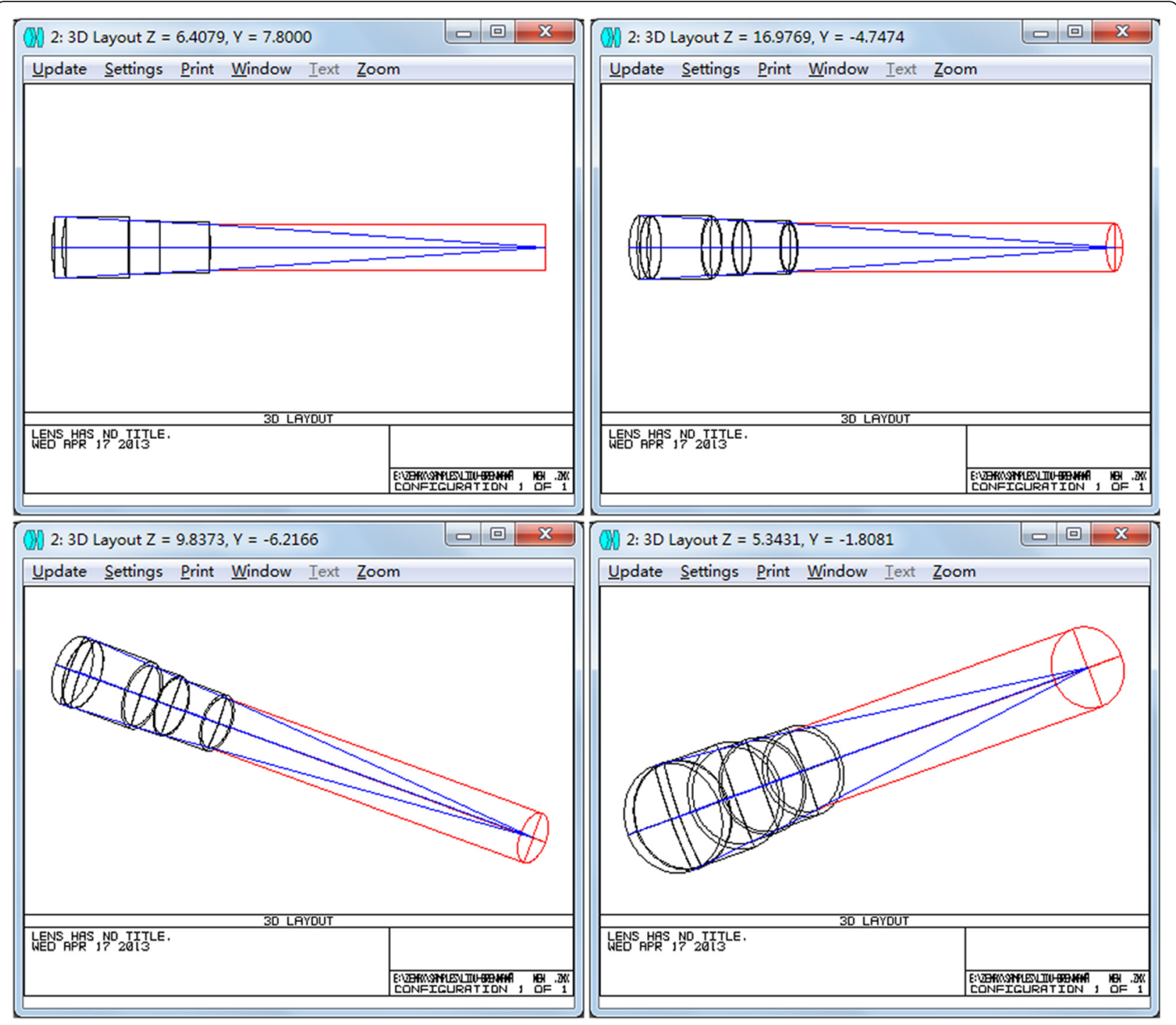

Fig. 2 3D stimulated light pathways of Liou-Brennan eye models

observed that the MTF values increased with IOL diopters, as measured under monochromatic light (Fig. 4b).

\section{Discussion}

\section{Parameters of IOL eye model}

The pupil plays an important role in optical imaging [14]. Studies $[3,4,15,16]$ have demonstrated that correcting spherical and chromatic aberrations simultaneously could increase visual acuity and contrast sensitivity under white light as well as provide better visual quality with a broader range of pupil diameters. Decreased visual quality was closely associated with increased spherical aberration of IOLs after the implantation of traditional spherical IOLs [17]; however, a diameter of the pupil of $3 \mathrm{~mm}$ is very close to the pupil diameter in diffraction-limited systems (pupil diameter less than $2.5 \mathrm{~mm}$ ), indicating that the influence from chromatic aberration is even greater than other factors, compared with eyes with their own optical aberrations. Campbell and Gubisch reported that whereas for $4 \mathrm{~mm}$ pupils the effect of spherical and chromatic aberration was approximately equal, monochromatic aberrations had a larger effect for large pupils [18]. In the present study, the differences between the MTF values for pupils with diameters less than $3 \mathrm{~mm}$ that were measured under monochromatic and polychromatic light were found to increase with diopters, which could have been caused mainly by chromatic aberration. The IOL material and its unique dependency between refractive index and wavelength are of critical importance for the optical performance of IOLs in polychromatic light [19]. As chromatic aberration is associated with the material of 

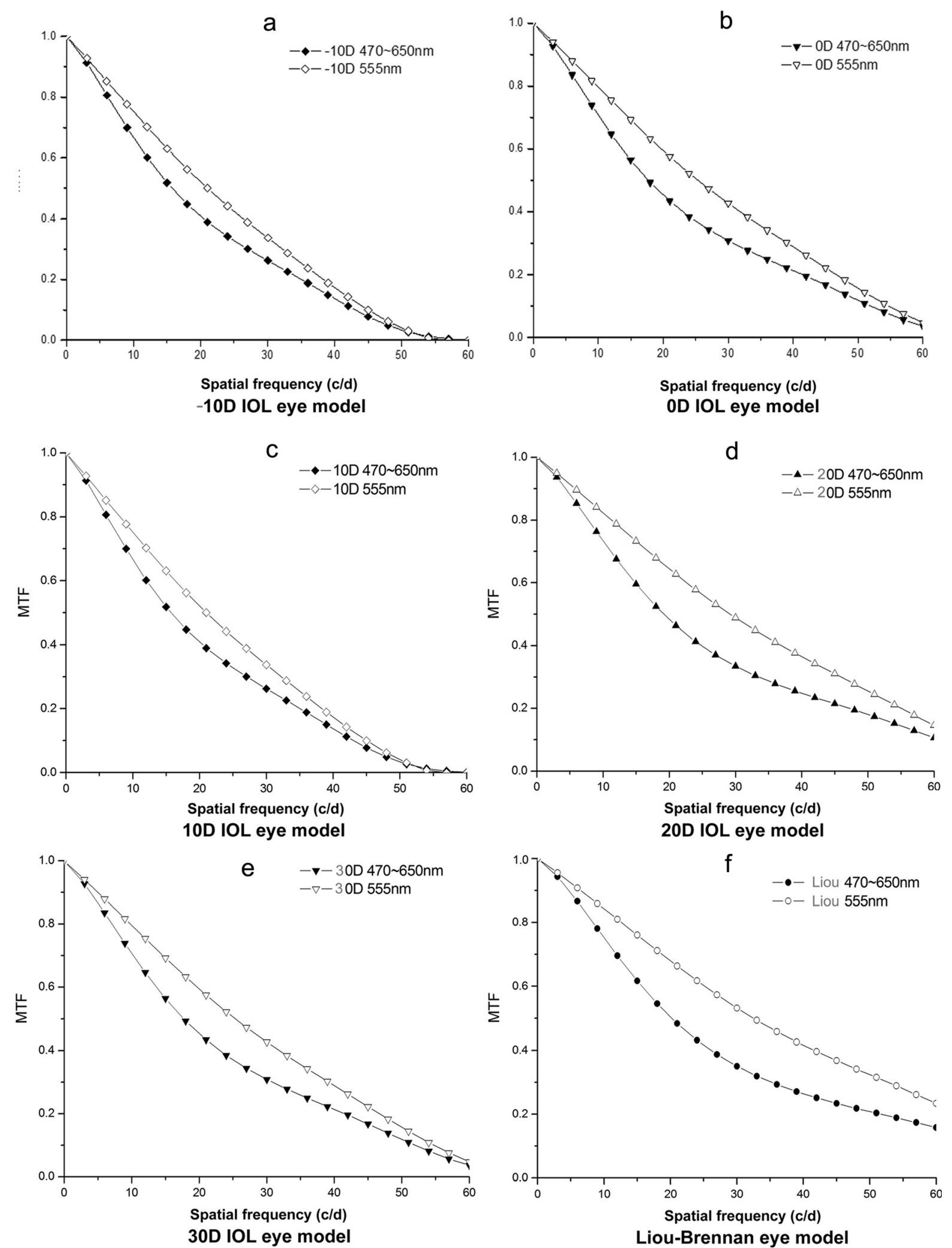

Liou-Brennan eye model

Fig. 3 Modulation transfer function (MTF) curves of the IOLs from -10 diopters (D) to $30 \mathrm{D}$ and Liou-Brennan eye models under monochromatic and polychromatic light. a. -10 D IOL eye model, b. 0 D IOL eye model, c. 10 D IOL eye model, d. 20 D IOL eye model, e. 30 D IOL eye model, f. Liou-Brennan eye model 
Table 3 A-value of the IOL eye models with different diopters at $3 \mathrm{~mm}$ of the pupil diameter

\begin{tabular}{llllll}
\hline $\begin{array}{l}\text { SF } \\
(\mathrm{c} / \mathrm{d})\end{array}$ & $30 \mathrm{D}$ & $20 \mathrm{D}$ & $10 \mathrm{D}$ & $\mathrm{OD}$ & $-10 \mathrm{D}$ \\
\hline 3 & 1.241 & 1.285 & 1.353 & 1.449 & 1.595 \\
6 & 4.495 & 4.595 & 4.752 & 4.991 & 5.344 \\
9 & 8.951 & 9.077 & 9.270 & 9.556 & 9.923 \\
12 & 13.909 & 14.019 & 14.128 & 14.271 & 14.341 \\
15 & 19.031 & 18.892 & 18.768 & 18.548 & 17.954 \\
24 & 31.353 & 30.192 & 28.620 & 26.333 & 22.675 \\
33 & 37.830 & 35.336 & 32.098 & 27.644 & 21.559 \\
42 & 40.159 & 36.569 & 31.629 & 25.449 & 21.245 \\
51 & 40.562 & 35.455 & 28.944 & 24.503 & 11.747 \\
60 & 39.331 & 32.501 & 27.721 & 23.957 & - - \\
\hline
\end{tabular}

$I O L$ intraocular lens, SF spatial frequencies, MTF modulation transfer function, $D$ diopter, $A$ value $=\Delta M T F / M T F_{1} ; \Delta M T F=M T F_{1}-M_{T F} ; M T F_{1}$ represents the MTF values measured under $555 \mathrm{~nm}$ of monochromatic light, $\mathrm{MTF}_{2}$ represents the MTF values measured under $470-650 \mathrm{~nm}$ of polychromatic light

the IOL, in the present study, only AR40e IOLs were chosen because they were the same type and were designed with the same materials.

Retinal image quality in the human eyes is dependent on the features of neural pathways. Studies have demonstrated that the cut-off spatial frequency is approximately $60 \mathrm{c} / \mathrm{d}$ under photopic conditions [20]; however, when the spatial frequency was increased to $32 \mathrm{c} / \mathrm{d}$, some healthy adults could not detect the gratings, suggesting that they had very low contrast sensitivity [21]. $\mathrm{Qu}$ et al. also demonstrated that MTF could be used to reflect the contrast sensitivity of the optical system of the eyes [22]. The MTF values of the eyes of healthy adults are very low at a spatial frequency of $32 \mathrm{c} / \mathrm{d}$ and thus could provide very limited value for clinical investigations. Lower spatial frequencies could affect outline identification more than higher spatial frequencies, and most of the information that is received by the eyes is from low spatial frequencies [23]. Because excellent retinal image contrast is also required under low spatial frequencies, in addition to higher cut-off spatial frequencies [24], 0-60 c/d of the spatial frequencies were chosen for the investigation in the present study.

The retina is most sensitive to yellow light with a wavelength of $555 \mathrm{~nm}$ under photopic conditions [25]; while for polychromatic light, we chose light with wavelengths ranging from 470 to $650 \mathrm{~nm}$ (including monochromatic light with wavelengths of $470,510,555,610$, and $650 \mathrm{~nm}$ and with weight of $0.091,0.503,1,0.503$, and 0.107 , respectively). The MTF values were measured under monochromatic and polychromatic light and were compared to investigate the effects of chromatic aberration on retinal image qualities.

\section{Visual qualities of the IOL eye models}

In the present study, we investigated the effects of IOLs on the chromatic aberrations of human eyes with different refraction states. The findings demonstrated that the MTF values were significantly lower when measured under polychromatic light than when measured under monochromatic light for each of the IOLs, without influences from other factors, suggesting that chromatic aberrations could reduce retinal image quality. Polychromatic MTF contains information on both monochromatic and chromatic aberrations [26]. While the monochromatic MTF of the eye clearly exceeds the polychromatic MTF and there is evidence that, in the absence of both chromatic and monochromatic aberrations, visual performance exceeds that
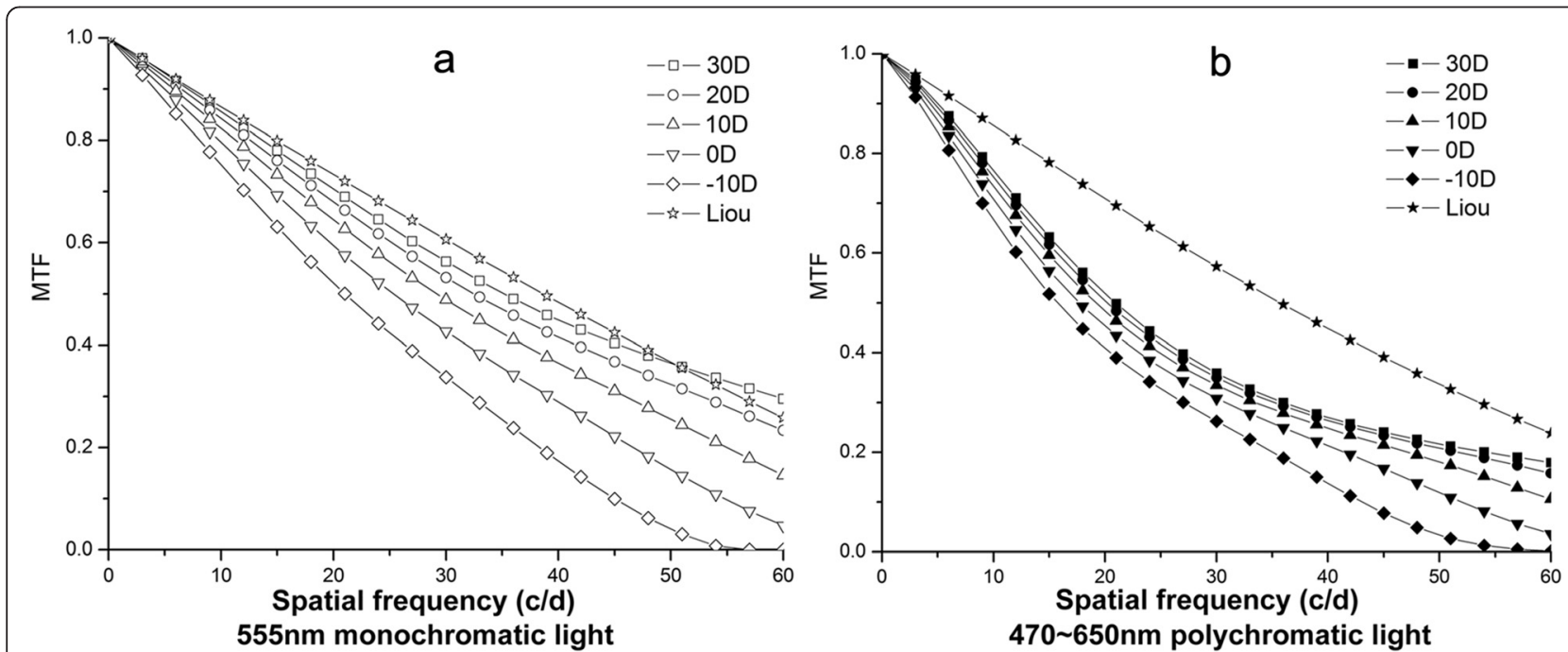

Fig. 4 Modulation transfer function (MTF) curves of the IOL from -10 diopters (D) to $30 \mathrm{D}$ and Liou-Brennan eye models under monochromatic and polychromatic light. a. $555 \mathrm{~nm}$ monochromatic light, b. $470-650 \mathrm{~nm}$ polychromatic light 
with noncorrected chromatic aberrations [8, 27, 28]. In addition, we also found that the difference between the MTF values that were measured under monochromatic and polychromatic light increased with the diopters of the IOLs (indicating that the central thickness of the IOLs increased), suggesting the increased influences of chromatic aberrations on retinal image quality. The findings of the present study also suggested that shorter axial length required IOLs with higher diopters, which could result in higher chromatic aberrations. Therefore, for patients with short axial length who require IOLs with high diopters, IOLs with low chromatic aberration should be selected to improve the postoperative visual quality.

However, with the increase in IOL diopters, the influence of chromatic aberrations on the MTF values decreased when the spatial frequencies were $\leq 12 \mathrm{c} / \mathrm{d}$ for pupils with diameters of $3 \mathrm{~mm}$, which could also reflect the effects of chromatic aberrations on the retinal image quality decreasing at medium or low spatial frequency when IOL with higher central thickness was used. Generally, most of the information that is received by the eyes is from low spatial frequencies, and lower spatial frequencies could affect outline identification more than higher spatial frequencies [23]. In another study performed by Nio et al., the findings showed that the contrast sensitivity of normal human eyes peaked at medium and low spatial frequencies $(4-8 \mathrm{c} / \mathrm{d})$ [21]. Therefore, for elderly patients undergoing cataract extraction and IOL implantation, IOLs with higher diopters could reduce the effects of chromatic aberrations on retinal image quality while identifying the outlines of objects, whereas IOLs with lower diopters could increase the effects.

The findings of the present study demonstrated that the MTF values of all of the IOL eye models were lower than those of the Liou-Brennan eye models, under either monochromatic or polychromatic light, suggesting that the implantation of IOLs could reduce the retinal image quality of human eyes $[8,29]$, although the difference was not that significant for the "common" implanted IOL-powers (from $15 \mathrm{D}$ to $25 \mathrm{D}$ ). The differences between the MTF values in the IOL eye models and the Liou-Brennan eye models were not as high under polychromatic light than under monochromatic light $(555 \mathrm{~nm})$, suggesting that the introduction of chromatic aberrations could further decrease retinal image quality. Interestingly, we found that the MTF values increased with the IOL diopters, either under $555 \mathrm{~nm}$ of monochromatic light or under polychromatic light, which could have been caused by the interactions of monochromatic aberrations and chromatic aberrations in the eye models. In addition, the axial length of the eye model could change after the implantation of IOLs, as well as optimization of optical aberrations and out-of- focus images, which could in turn affect the MTF values. Moreover, with the increase in diopters of the IOLs, the central thickness also increased. Because the thicknesses of IOLs with higher diopters are closer those in the Liou-Brennan eye models $(4.02 \mathrm{~mm})$ with the increased IOL diopters, the MTF values of the IOL eye models could also approach the MTF values of the LiouBrennan eye models. However, no studies have investigated the detailed mechanisms, and further studies are warranted to validate the above-mentioned theories.

\section{Conclusion}

The present study showed that IOLs with different diopters affected chromatic aberrations of the eye when the diameter of the pupil was $3 \mathrm{~mm}$. The chromatic aberrations increased when IOLs with higher diopters (which also indicates higher central thickness) were implanted, thus reducing retinal image quality. The implantation of IOLs with relative high diopters for patients with less axial depth created relatively high chromatic aberrations, which could influence the visual quality of the patients. Interestingly, the increase in IOL diopters was mirrored by the decrease in the influence of the chromatic aberrations on visual quality at medium or low spatial frequencies. However, contradictory results were found at high spatial frequencies. The visual quality of the IOL eye model further decreased due to the effects of chromatic aberrations. The findings of the present study provided evidence for the designing of IOLs in the near future.

\section{Abbreviations \\ IOLs: intraocular lenses; D: diopter; MTF: modulation transfer function; \\ SF: spatial frequencies; LCA: longitudinal chromatic aberration; SA: spherical} aberration.

Competing interests

The authors declare that they have no competing or financial interests.

Authors' contributions

HS has been involved in designing the study and acquisition of the most data, and drafting the manuscript of this study. XY made contributions in adjusting the experimental designs, analyzing the results and revising the manuscript. XT supervised the study designs, analyised and interpreted the results, and did extensive contribution to the manuscript draft. All authors read and approved the final manuscript.

\section{Acknowledgments}

This study was supported by the Key Projects of the Bureau of Health, Tianjin (2012KR17, 10KG108).

Received: 30 August 2014 Accepted: 6 January 2016

Published online: 11 January 2016

References

1. Vinas M, Dorronsoro C, Cortes D, Pascual D, Marcos S. Longitudinal chromatic aberration of the human eye in the visible and near infrared from wavefront sensing, double-pass and psychophysics. Biomed Opt Express. 2015;6(3):948-62.

2. Artal P, Manzanera S, Piers $P$, Weeber H. Visual effect of the combined correction of spherical and longitudinal chromatic aberrations. Opt Express. 2010;18:1637-48. 
3. Weeber HA, Piers PA. Theoretical performance of intraocular lenses correcting both spherical and chromatic aberration. J Refract Surg. 2012;28:48-52

4. Sabesan R, Zheleznyak L, Yoon G. Binocular visual performance and summation after correcting higher order aberrations. Biomed Opt Express. 2012;3:3176-89.

5. Applegate RA, Thibos LN, Hilmantel G. Optics of aberroscopy and super vision. J Cataract Refract Surg. 2001;27:1093-107.

6. Weeber HA, Pohl R, Mester U, Piers PA. Visual performance of pseudophakic eyes corrected for spherical and chromatic aberrations with an achromatic intraocular lens. Invest Ophthalmol Vis Sci. 2013;54:808.

7. Schwarz C, Canovas C, Manzanera S, Weeber H, Prieto PM, Piers P, et al. Binocular visual acuity for the correction of spherical aberration in polychromatic and monochromatic light. J Vis. 2014;14:1-11.

8. Perez-Merino P, Dorronsoro C, Llorente L, Duran S, Jimenez-Alfaro I, Marcos S. In vivo chromatic aberration in eyes implanted with intraocular lenses. Invest Ophthalmol Vis Sci. 2013;54:2654-61.

9. Siedlecki D, Ginis HS. On the longitudinal chromatic aberration of the intraocular lenses. Optom and Vis Sci. 2007;84:984-9.

10. López-Gil N, Bradley A. The potential for and challenges of spherical and chromatic aberration correction with new IOL designs. Br J Ophthalmol. 2013;97(6):677-8.

11. Liou HL, Brennan NA. Anatomically accurate, finite model eye for optical modeling. J Opt Soc Am A Opt Image Sci Vis. 1997;14:1684-95.

12. Piers PA, Weeber HA, Artal P, Norrby S. Theoretical comparison of aberration-correcting customized and aspheric intraocular lenses. J Refract Surg. 2007:23:374-84.

13. Liang J, Williams DR. Aberration and retinal image quality of the normal human eye. J Opt Soc Am A Opt Image Sci Vis. 1997;14:2873-83.

14. Thibos LN, Bradley A, Still DL, Zhang X, Howarth PA. Theory and measurement of ocular chromatic aberration. Vision Res. 1990;30:33-49.

15. Awwad ST, El-Kateb M, McCulley JP. Comparative higher-order aberration measurement of the LADARWave and Visx WaveScan aberration at varying pupil sizes and after pharmacologic dilation and cycloplegia. J Cataract Refract Surg. 2006;32:203-14

16. Ravikumar S, Bradley A, Thibos LN. Chromatic aberration and polychromatic image quality with diffractive multifocal intraocular lenses. J Cataract Refract Surg. 2014;40(7):1192-204.

17. Guirao A, Redondo M, Arta LP. Optical aberrations of the human cornea as a function of age. J Opt Soc Am A Opt Image Sci Vis. 2000;17:1697-702.

18. Campbell FW, Gubisch RW. The effect of chromatic aberration on visual acuity. J Physiol. 1967;192:345-58.

19. Siedlecki D, Jozwik A, Zajac M, Hill-Bator A, Turno-Krecicka A. In vivo longitudinal chromatic aberration of pseudophakic eyes. Optom Vis Sci. 2014:91:240-6

20. Sekiguchi N, Williams DR, Brainard DH. Efficiency in detection of isoluminant and isochromatic interference fringes. J Opt Soc Am A Opt Image Sci Vis. 1993;10:2118-33

21. Nio YK, Jansonius NM, Fidler V, Geraghty E, Norrby S, Kooijman AC. Agerelated changes of defocus-specific contrast sensitivity in healthy subjects. Opthal Physiol Opt. 2000;20:323-34

22. Qu J, Lv F, Mao X. Evaluation of visual correction. Chin J Ophthalmol. 2003;6:325-7.

23. Wang Y, Zhao K. Wavefront Aberration and Clinical Vision Correction. Beijing: People's Medical Publishing House; 2011.

24. Kornowski JA, Petersik JT. Effect on face recognition of spatial-frequency information contained in inspection and test stimuli. J Gen Psychol. 2003;130:229-44

25. Zhao H, Mainster MA. The effect of chromatic dispersion on pseudophakic optical performance. Br J Ophthalmol. 2007:91:1225-9.

26. Williams CS, Becklund OA. Introduction to the optical transfer function. Bellingham: SPIE Press; 1989

27. McLellan JS, Marcos S, Prieto PM, Burns SA. Imperfect optics may be the eye's defense against chromatic blur. Nature. 2002;417:174-6.

28. Ravikumar S, Thibos LN, Bradley A. Calculation of retinal image quality for polychromatic light. J Opt Soc Am A Opt Image Sci Vis. 2008;25:2395-407.

29. Franchini A. Compromise between spherical and chromatic aberration and depth of focus in aspheric intraocular lenses. J Cataract Refract Surg. 2007:33:497-509.

\section{Submit your next manuscript to BioMed Central and we will help you at every step:}

- We accept pre-submission inquiries

- Our selector tool helps you to find the most relevant journal

- We provide round the clock customer support

- Convenient online submission

- Thorough peer review

- Inclusion in PubMed and all major indexing services

- Maximum visibility for your research

Submit your manuscript at www biomedcentral com/submit
Biomed Central 